\title{
Accretion disk in the binary system V367 Cygni`
}

\author{
S. Zoła ${ }^{1,2}$ and W. Ogłoza ${ }^{2,3}$ \\ 1 Astronomical Observatory of the Jagiellonian University, ul. Orla 171, 30-244 Cracow, Poland \\ 2 Mt. Suhora Observatory of the Pedagogical University, ul. Podchorążych 2, 30-084 Cracow, Poland \\ 3 N. Copernicus Astronomical Center, Polish Academy of Sciences, ul. Bartycka 18, 00-716 Warsaw, Poland \\ Received 4 April 2000/ Accepted 2 January 2001
}

\begin{abstract}
New photoelectric observations of the interacting binary V367 Cyg were made during two consecutive seasons, 1996 and 1997, using the two-channel photometer at Mt. Suhora Observatory. The BVRI light curves are analyzed and system parameters are derived for two alternative models: with and without an accretion disk. A contact configuration is obtained for the no-disk model. The semidetached model, with a disk around the invisible component gives a better fit and, in addition, explains most of the observed features of V367 Cyg. The disk in V367 Cyg has a radius of about $R_{\mathrm{d}}=23 R_{\odot}$, almost completely filling the secondary component's Roche lobe. Mass is transferred from the less massive $\left(3.3 M_{\odot}\right)$ to the more massive $\left(4.0 M_{\odot}\right)$ star at a high rate of $5^{-7} 10^{-5} M_{\odot} / \mathrm{yr}$.
\end{abstract}

Key words. binaries: eclipsing, accretion disks, individual: V367 Cyg

\section{Introduction}

V367 Cyg $\left(\mathrm{BD}+38^{\circ} 4235, V=7.04^{\mathrm{m}}, B=7.66^{\mathrm{m}}\right.$, $P=18$. 6, Sp $=$ A7Iapevar, RA $=20^{\mathrm{h}} 47^{\mathrm{m}} 59.6$, DEC $=$ $+39^{\circ} 17^{\prime} 15^{\prime \prime} .7(\mathrm{~J} 2000)$ ), is an eclipsing system that belongs to the Serpentids group. Several photometric light curves have been published, mostly obtained in the $U B V$ system (Heiser 1961; Fresa 1966; Kalw \& Pustylnik 1975; Akan 1987). The authors observed variations in depth of both minima as well as intrinsic fluctuations in the light curves. In addition, an asymmetry of both minima was noticed.

V367 Cyg is a multiple system. Two optical companions were reported: $\mathrm{C}$, which is faint $(13.7 \mathrm{mag})$ and about 2 arcsec distant from V367 Cyg and D (HD 198288), supposed to be only 0.2 arcsec away but of comparable brightness. The D component was at first resolved and then undetected by the speckle technique (McAlister \& Hartkopf 1988) and some authors questioned its existence. Most of the recent work on V367 Cyg does not include star D in the model of the system.

Only the primary component is observed spectroscopically. The underlying stellar spectrum is dominated by strong, sharp shell lines mostly of singly-ionized metals. Emission lines of highly-ionized elements such as NV, CIV and SiIV were also detected (Heiser 1962; Aydin et al. 1978). MgII $\lambda 4481$ is the only clearly visible photospheric

\footnotetext{
Send offprint requests to: S. Zoła,

e-mail: sfzola@cyf-kr.edu.pl

* The original observations are available in electronic form at the CDS via anonymous ftp to cdsarc.u-strasbg.fr (130.79.128.5) or via

http://cdsweb.u-strasbg.fr/cgi-bin/qcat?J/A+A/368/932
}

line. Other lines belonging to the primary component were noticed as blends or asymmetries of the shell lines. Comparing the photospheric wings of the Balmer lines with models computed by Kurucz, Aydin et al. (1978) derived the primary star's temperature $T_{\text {eff }}=8000 \mathrm{~K}$ and surface gravity $\log g=1.5$.

Although Glazunova \& Menchenkova (1989) announced the detection of the MgII $\lambda 4481$ line originating in the secondary star, this interpretation has been ruled out by Schneider et al. (1993). Based on new CCD data, they attributed the proposed secondary's line to the FeI $\lambda 4482.2$ shell line. Unfortunately, this result leaves the mass ratio of the system still not determined from the radial velocity curves.

The light curve of V367 Cyg was modeled by Li \& Leung (1987), who found a contact configuration for this system. However, in a more recent paper, Pavlovski et al. (1992) have shown that the light curve can also be described quite well by a completely different geometry, namely the semidetached configuration. From the light curve synthesis, the authors derived a solution with a mass ratio $q=0.56$ (primary/secondary) and inclination $i=81.4^{\circ}$. In this model, the enigmatic secondary component is an accretion disk hiding the mass gaining star $\left(T_{\mathrm{s}}=13500 \mathrm{~K}\right)$. Within the model proposed by Pavlovski et al. (1992), the disk is represented by a slab of vertical thickness $z_{\mathrm{d}}=0.041 \mathrm{~A}$, radius $r_{\mathrm{d}}=0.43 \mathrm{~A}$ and uniform temperature distribution.

Low resolution spectra made by IUE were analyzed by Hack et al. (1984). They found that the system is heavily reddened, with $E(B-V)$ between 0.50 and 0.55 . By comparing the dereddened flux in the ultraviolet 
continuum with Kurucz (1979) models, it turned out that one star is not sufficient to explain the observed distribution. The best fit was obtained assuming two stars, with temperatures $8000 \mathrm{~K}(\log g=1.5)$ and $10000 \mathrm{~K}$. Contrary to expectations based on BV photometry, the secondary component is hotter than the primary one. Hack et al. proposed that the eclipsing body is a cool disk around the secondary star with $T_{\text {eff }}=10000 \mathrm{~K}$.

Polarimetric observations of V367 Cyg have been published by Elias (1993). He constructed a two-component model for the circumbinary matter. The variable linear polarization component arises from an electron envelope around the stars. Constant linear component as well as variable circular polarization are produced by an extensive envelope of material consisting of neutral hydrogen and dust surrounding the whole system. Recently Berdyugin \& Tarasov (1997) found, contrary to the results by Elias, that the circular polarization (within the observational errors) was zero. They also argue that the intrinsically variable polarization probably results from light scattering by a gas stream that flows from the primary to the secondary through the inner Lagrangian point. The analysis of the polarization variability gave the orbital inclination to be roughly 82 degrees.

The most recent model of the interacting binary V367 Cyg was proposed by Tarasov \& Berdyugin (1998) (TB98) based on new, high-dispersion CCD spectroscopic observations. TB98 have determined radial velocities of the primary component from the MgII $\lambda 4481$ line at several phases. Combining them with previously published radial velocities, TB98 confirmed long-period variations in systemic velocity $\gamma_{0}$ and derived a new value for the primary star semi-amplitude $K_{\mathrm{p}}=85 \pm 2 \mathrm{~km} \mathrm{~s}^{-1}$ (leading to a new value for the mass function $f(m)=1.19 M_{\odot}$ and a sini $\left.=32.5 R_{\odot}\right)$. The authors claim detection of a faint emission component of the HeI $\lambda 6678$ line. They argue that the HeI $\lambda 6678$ emission is formed in an accretion disk surrounding the invisible component and have used it to determine $K_{\mathrm{s}}=177 \pm 24 \mathrm{~km} \mathrm{~s}^{-1}$, and, consequently $M_{\mathrm{p}}=22 M_{\odot}, M_{\mathrm{s}}=11 M_{\odot}$. An inspection of their Fig. 1 shows, however, that there is no distinct feature in the complex line profile that could be reliably identified as a "component". TB98 concluded that V367 Cyg is in an initial phase of rapid mass transfer (as previously suggested by Paczyński 1971), with matter flowing from the more massive to the less massive component.

In this paper we present the analysis of new photometric light curves of V367 Cyg obtained in the BVRI filters. We describe the new observations in the next section. Description of modeling of the new photometric light curves is presented in Sect. 3, while the results are discussed in the last section.

\section{New observations}

We decided to re-observe this star in order to obtain a complete light curve in the shortest possible time, with the hope of reducing intrinsic variations of the light curve. In

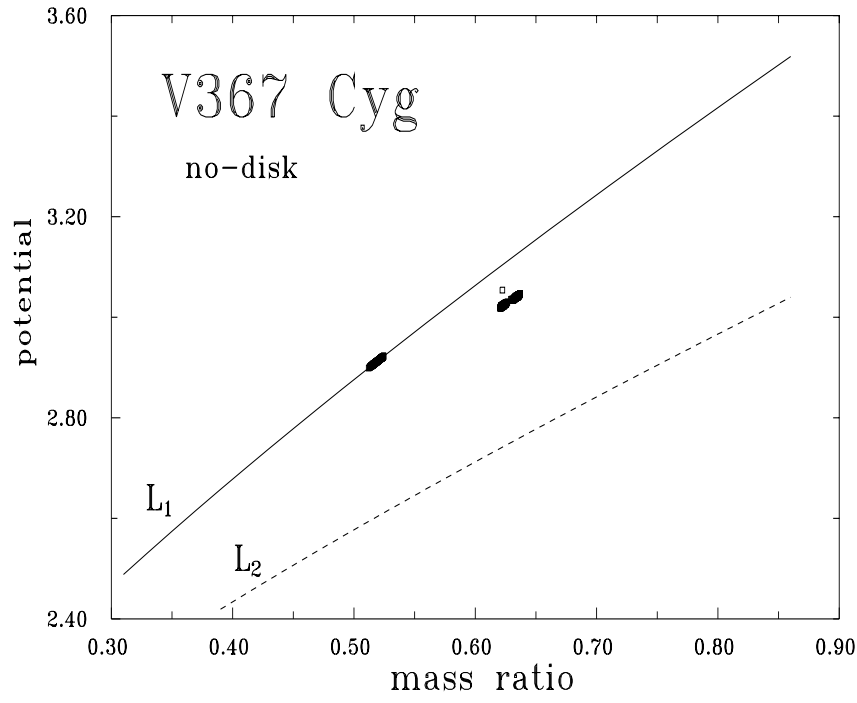

Fig. 1. Obtained configuration of V367 Cyg in the no-disk model. The lines show potentials $\Omega$ at the Lagrangian points $L_{1}$ (continuous) and $L_{2}$ (dashed) as a function of mass ratio. Points show cross-section of the search arrays in the $\Omega-q$ plane at the end of search for the two solutions considered: with third light ( $q$ close to 0.62 ) and without third light ( $q$ close to 0.51 )

addition, we wanted to extend photometry to the $R$ and $I$ bands.

V367 Cyg was observed with the $60 \mathrm{~cm}$ telescope and two-channel photometer at the Mt. Suhora Observatory. A description of the equipment was published by Kreiner et al. (1993). We used $\mathrm{BD}+38^{\circ} 4242$ as the comparison star and $\mathrm{BD}+38^{\circ} 4239$ as the check star. Data were collected during two consecutive seasons in 1996 and 1997. During this time, we succeeded in obtaining complete light curves in the $B V R I$ filters. All data were reduced in the usual way. First we cross-calibrated both channels for different sensitivity; next, the correction for differential extinction was applied. The data, magnitude differences between the variable and comparison stars, was left in the instrumental system. The phases were calculated using the ephemeris published by Pavlovski et al. (1992).

Large scatter (which for such a bright star cannot be explained by observational errors) is visible also in our data throughout all phases. However, its amplitude slightly decreases towards longer wavelengths. An asymmetry of the secondary minimum is also present in the new observations.

\section{Light curve modeling}

\subsection{No-disk model}

Since V367 Cyg is a multiple system, we initially limited the solutions to these which include a third light in the list of free parameters.

Assuming that the system consists of only stellar components, our first attempt was to find the best solution using the Wilson-Devinney code (Wilson \& Devinney 1971; Wilson 1993). The Monte Carlo method (Price 1976; 
Table 1. Results derived from light curve modeling within the no-disk model

\begin{tabular}{|c|c|c|}
\hline parameter & with $l_{3}$ & without $l_{3}$ \\
\hline configuration & contact & near - contact \\
\hline phase shift & $-0.0009 \pm 0.0001$ & $-0.0005 \pm 0.0001$ \\
\hline i & $87^{\circ} 4 \pm 0.4$ & $80^{\circ} 3 \pm 0.3$ \\
\hline$T_{\mathrm{p}}$ & $* 8000 \mathrm{~K}$ & $* 8000 \mathrm{~K}$ \\
\hline$T_{\mathrm{s}}$ & $6730 \pm 25 \mathrm{~K}$ & $6500 \pm 30 \mathrm{~K}$ \\
\hline$\Omega_{\mathrm{p}}$ & $3.008 \pm 0.007$ & $2.888 \pm 0.009$ \\
\hline$\Omega_{\mathrm{s}}$ & $* * 3.008$ & $2.888 \pm 0.014$ \\
\hline$q\left(M_{\mathrm{s}} / M_{\mathrm{p}}\right)$ & $0.620 \pm 0.011$ & $0.507 \pm 0.027$ \\
\hline$L_{\mathrm{p}}(B)$ & $7.006 \pm 0.024$ & $9.965 \pm 0.025$ \\
\hline$L_{\mathrm{p}}(V)$ & $6.898 \pm 0.019$ & $9.642 \pm 0.020$ \\
\hline$L_{\mathrm{p}}(R)$ & $6.780 \pm 0.015$ & $9.419 \pm 0.016$ \\
\hline$L_{\mathrm{p}}(I)$ & $6.859 \pm 0.012$ & $9.105 \pm 0.013$ \\
\hline$L_{\mathrm{s}}(B)$ & $* * 2.190$ & $* * 2.193$ \\
\hline$L_{\mathrm{s}}(V)$ & $* * 2.412$ & $* * 2.473$ \\
\hline$L_{\mathrm{s}}(R)$ & $* * 2.563$ & $* * 2.651$ \\
\hline$L_{\mathrm{s}}(I)$ & $* * 2.870$ & $* * 2.834$ \\
\hline$l_{3}(B)$ & $0.229 \pm 0.008$ & $*_{0.000}$ \\
\hline$l_{3}(V)$ & $0.218 \pm 0.007$ & $* 0.000$ \\
\hline$l_{3}(R)$ & $0.212 \pm 0.006$ & $*_{0} 0.000$ \\
\hline$l_{3}(I)$ & $0.178 \pm 0.005$ & $* 0.000$ \\
\hline$L_{\mathrm{p}} /\left(L_{\mathrm{p}}+L_{\mathrm{s}}\right)(B)$ & 0.762 & 0.820 \\
\hline$L_{\mathrm{p}} /\left(L_{\mathrm{p}}+L_{\mathrm{s}}\right)(V)$ & 0.741 & 0.796 \\
\hline$L_{\mathrm{p}} /\left(L_{\mathrm{p}}+L_{\mathrm{s}}\right)(R)$ & 0.726 & 0.780 \\
\hline$L_{\mathrm{p}} /\left(L_{\mathrm{p}}+L_{\mathrm{s}}\right)(I)$ & 0.705 & 0.763 \\
\hline$L_{\mathrm{s}} /\left(L_{\mathrm{p}}+L_{\mathrm{s}}\right)(B)$ & 0.238 & 0.180 \\
\hline$L_{\mathrm{s}} /\left(L_{\mathrm{p}}+L_{\mathrm{s}}\right)(V)$ & 0.259 & 0.204 \\
\hline$L_{\mathrm{s}} /\left(L_{\mathrm{p}}+L_{\mathrm{s}}\right)(R)$ & 0.274 & 0.220 \\
\hline$L_{\mathrm{s}} /\left(L_{\mathrm{p}}+L_{\mathrm{s}}\right)(I)$ & 0.295 & 0.237 \\
\hline$\chi^{2}$ (weighted) & 4.373 & 4.399 \\
\hline
\end{tabular}

Barone et al. 1988; Zoła et al. 1997) was employed as the search procedure instead of the original differential corrections (DC). The Monte Carlo method can locate a global minimum within the set ranges of parameters.

Normal points were calculated from the original observations: 78 in the $I$ filter and 85 in the $B, V$ and $R$ filters. We applied the following weighting scheme for our data. The $V$ and $R$ light curves were considered more accurate and assigned weights twice larger than those of the $B$ and $I$ data. Additionally, in each light curve points in the deeper minimum have larger weights than those outside.

The temperature of the primary component (star No. 1 in the W-D model), which is observed spectroscopically, was fixed at $8000 \mathrm{~K}$. The limb darkening coefficients were adopted as functions of the temperature and wavelength from Díaz-Cordovés et al. (1995) and Claret et al. (1995).
Additionally, we set albedos and gravity darkening coefficients at their theoretical values. The following parameters were adjusted: the phase shift, inclination, temperature of the secondary component, potentials of the stars, the mass ratio, luminosity of the primary and a third light. We used a multidimensional search array consisting of 2000 elements. Each element consists of all parameters needed for computing a synthetic light curve and an additional parameter describing the quality of the fit. Initially, the search array was filled with randomly chosen parameters from assumed ranges. In subsequent computations, each successive trial produced a new set of parameters which was compared with the worst one stored in the search array. If its quality was better than that of the worst element, the latter was replaced in the search array. This procedure was continued until the difference between the best and the worst elements of the search array were smaller than 1 percent. The computations were repeated in the same way as described above but assuming $l_{3}=0$. Final results are presented in Table 1 . The errors listed in Table 1 are standard $\sigma$ 's computed from all values of each adjusted parameter stored the search array.

The results are also shown graphically in Fig. 1. In this figure, the cross-sections of the search arrays in the $\Omega-q$ plane are shown for both cases considered. The two clouds of points shown in Fig. 1 represent the best solutions stored in the search arrays at the end of search. As one can see, the contact configuration was obtained for the model which included a third light and near-contact if $l_{3}=0$.

\subsection{Disk model}

Next, we considered a semidetached model with an accretion disk. Such a solution would explain some observed properties of V367 Cyg not understood within the model described in the previous section, i.e. the presence of highly-ionized, metal lines or invisibility of the spectrum of the hotter star. The model is based on the WD code, modified to include an optically thick disk (Zoła 1991, 1992). It requires four parameters attributed to the disk: its radius $\left(R_{\mathrm{d}}\right)$, luminosity $\left(l_{\mathrm{d}}\right)$, outer temperature $\left(T_{\text {out }}\right)$ (in this paper, temperature distribution is assumed as for the stationary-accretion case), and thickness $(\beta)-$ i.e. the angle between the disk surface and the orbital plane. The disk thickness is assumed to increase linearly with radial distance, which can be a first order approximation for moderately thick disks. In our model, the accretion disk surrounds the secondary star. The primary star is assumed to fill its Roche lobe. The deeper minimum in V367 Cyg would then be caused by the eclipse of the star whose spectrum is observed by an accretion disk.

Following Hack et al. (1984), we fixed the temperature of the primary (the mass loser) at $8000 \mathrm{~K}$. As in the no-disk model, we included a third light in the list of free parameters but also checked for a possible solution without third light. The linear limb darkening coefficients 
were taken from Díaz-Cordovés et al. (1995) and Claret et al. (1995), according the temperatures and filter wavelengths, and again we set albedos and gravity darkening coefficients equal to their theoretical values.

The normal mode of the W-D code operation is when the control parameter $I P B$ is set to 0 . For such a case, the relative luminosity of the secondary $\operatorname{star}\left(L_{\mathrm{s}}\right)$ is not a free parameter but is computed from geometrical parameters, the luminosity of the primary component, temperatures and a radiation law (either black body or CarbonGingerich atmosphere models). The IPB parameter can be set to 1 . Then the coupling between $\left(L_{\mathrm{s}}\right)$ and $\left(T_{\mathrm{s}}\right)$ is severed and both luminosities can be adjusted. If there is an optically thick disk in a binary system, then for high inclination, the disk can completely obscure the mass gaining component. Indeed after some trial runs we encountered just such a situation with V367 Cyg, and set the control parameter $I P B=1$. The following parameters were fitted: inclination, the mass ratio, luminosities of both stars, the secondary star potential, the disk parameters and the third light. The temperature of the secondary star was fixed at $10000 \mathrm{~K}$, after Hack et al. (1984). Computations were done until the difference between the best and the worst elements in the search array was less than 1 percent. Next, we switched to the gradient search algorithm, setting the initial values of the parameters to those for which the best fit was derived. The results are presented in Table 2. The errors listed in Table 2 are standard $\sigma$ 's computed for each free parameter from all elements of the search array. We derived a configuration where the disk completely obscures the mass gainer, for which there was no convergence either for the secondary star's potential or its relative luminosity $\left(L_{\mathrm{s}}\right)$ and there are no entries for these parameters in Table 2. Comparison between the synthetic and observed light curves is shown in Figs. 2 and 3 (the disk model with third light). One can see views of the disk model at phase 0.0 in Fig. 4.

Finally, we checked the model proposed by TB98. The computations were performed with the gradient search method only and with the IPB parameter set to 1 . The mass ratio parameter was fixed at $q=2.08$ (primary/secondary), the value derived by TB98. We also fixed the temperatures at $10000 \mathrm{~K}$ and $8000 \mathrm{~K}$ (for the secondary and primary, respectively) and inclination at 82 degrees. It turned out that a good agreement with the new data can be obtained only for very thick disks exceeding the Roche lobe of the secondary star.

\section{Discussion}

\subsection{Absolute dimensions}

Combining the results from this paper (no-disk and disk models) with the mass function $f(m)=1.19 M_{\odot}$ and a $\sin i=32.5 R_{\odot}$ (TB98), we can derive the absolute parameters of the components, presented in Table 3. Since the secondary star is completely obscured by the accretion disk we have no information about its radius if the disk
Table 2. Results derived from light curve modeling within the disk model

\begin{tabular}{|c|c|c|}
\hline parameter & with $l_{3}$ & without $l_{3}$ \\
\hline configuration & semidetached & semidetached \\
\hline phase shift & $* 0.0000$ & $* 0.0000$ \\
\hline $\mathrm{i}$ & $88^{\circ} 8 \pm 0.9$ & $84^{\circ} 8 \pm 0.9$ \\
\hline$T_{\mathrm{s}}$ & $* 10000 \mathrm{~K}$ & $* 10000 \mathrm{~K}$ \\
\hline$T_{\mathrm{p}}$ & $* 8000 \mathrm{~K}$ & $* 8000 \mathrm{~K}$ \\
\hline \multicolumn{3}{|l|}{$\Omega_{\mathrm{s}}$} \\
\hline$\Omega_{\mathrm{p}}$ & $* * 2.765$ & $* * 3.462$ \\
\hline$q\left(M_{\mathrm{p}} / M_{\mathrm{s}}\right)$ & $0.443 \pm 0.091$ & $0.826 \pm 0.120$ \\
\hline \multicolumn{3}{|l|}{$L_{\mathrm{s}}(B)$} \\
\hline \multicolumn{3}{|l|}{$L_{\mathrm{s}}(V)$} \\
\hline \multicolumn{3}{|l|}{$L_{\mathrm{s}}(R)$} \\
\hline \multicolumn{3}{|l|}{$L_{\mathrm{s}}(I)$} \\
\hline$L_{\mathrm{p}}(B)$ & $8.826 \pm 0.065$ & $9.898 \pm 0.037$ \\
\hline$L_{\mathrm{p}}(V)$ & $8.830 \pm 0.064$ & $9.716 \pm 0.027$ \\
\hline$L_{\mathrm{p}}(R)$ & $8.870 \pm 0.072$ & $9.548 \pm 0.024$ \\
\hline$L_{\mathrm{p}}(I)$ & $9.094 \pm 0.049$ & $9.336 \pm 0.031$ \\
\hline$l_{3}(B)$ & $0.091 \pm 0.005$ & $* 0.000$ \\
\hline$l_{3}(V)$ & $0.069 \pm 0.004$ & $* 0.000$ \\
\hline$l_{3}(R)$ & $0.047 \pm 0.005$ & $* 0.000$ \\
\hline$l_{3}(I)$ & $0.001 \pm 0.001$ & $* 0.000$ \\
\hline$R_{\mathrm{d}}$ & $0.382 \pm 0.003$ & $0.391 \pm 0.006$ \\
\hline$\beta$ & $16.0 \pm 2.1$ & $15.3 \pm 1.8$ \\
\hline$T_{\text {out }}$ & $5200 \mathrm{~K} \pm 700$ & $5300 \mathrm{~K} \pm 1550$ \\
\hline$l_{\mathrm{d}}(B)$ & $0.155 \pm 0.004$ & $0.173 \pm 0.004$ \\
\hline$l_{\mathrm{d}}(V)$ & $0.184 \pm 0.003$ & $0.193 \pm 0.003$ \\
\hline$l_{\mathrm{d}}(R)$ & $0.203 \pm 0.003$ & $0.204 \pm 0.003$ \\
\hline$l_{\mathrm{d}}(I)$ & $0.227 \pm 0.004$ & $0.210 \pm 0.003$ \\
\hline$\chi^{2}$ (weighted) & 4.245 & 4.581 \\
\hline
\end{tabular}

$*-$ not adjusted, $\quad * *-$ computed,

$L_{\mathrm{p}}, L_{\mathrm{s}}: \mathrm{W}-\mathrm{D}$ program input values.

model $(I P B=1)$ solution is considered. However, if we assume that this component is a Main Sequence star, for its mass of $M_{\mathrm{S}}=2.5 M_{\odot}$ its radius should be $R_{\mathrm{S}}=2.3 R_{\odot}$, while the temperature $T_{\mathrm{s}}=10400 \mathrm{~K}$ (Harmanec 1988). The solution without $l_{3}$ would result in the following parameters of the secondary component: $M_{\mathrm{s}}=4.0 M_{\odot}$, $R_{\mathrm{s}}=2.9 R_{\odot}$ and $T_{\mathrm{s}}=14800 \mathrm{~K}$.

The accretion disk has a radius of about $23 R_{\odot}$ and outer vertical thickness of about $6 R_{\odot}$. We noticed that convergence of the outer disk temperature parameter was poorer than for others. Therefore, the disk temperature (shown in Table 4) has been computed by comparing the vertical thickness of the disk resulting from modeling and computed from the $\alpha$-disk, $z$-structure models (Smak 1984, 1992). 

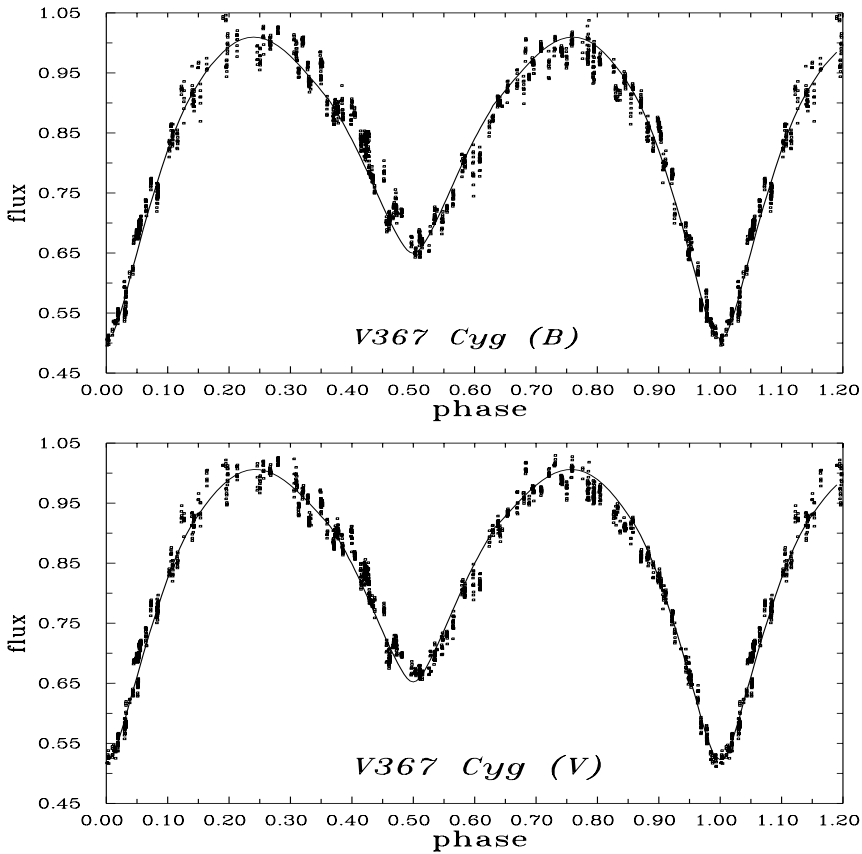

Fig. 2. Comparison between the disk model (lines) and observations (dots) $-B$ and $V$ filters

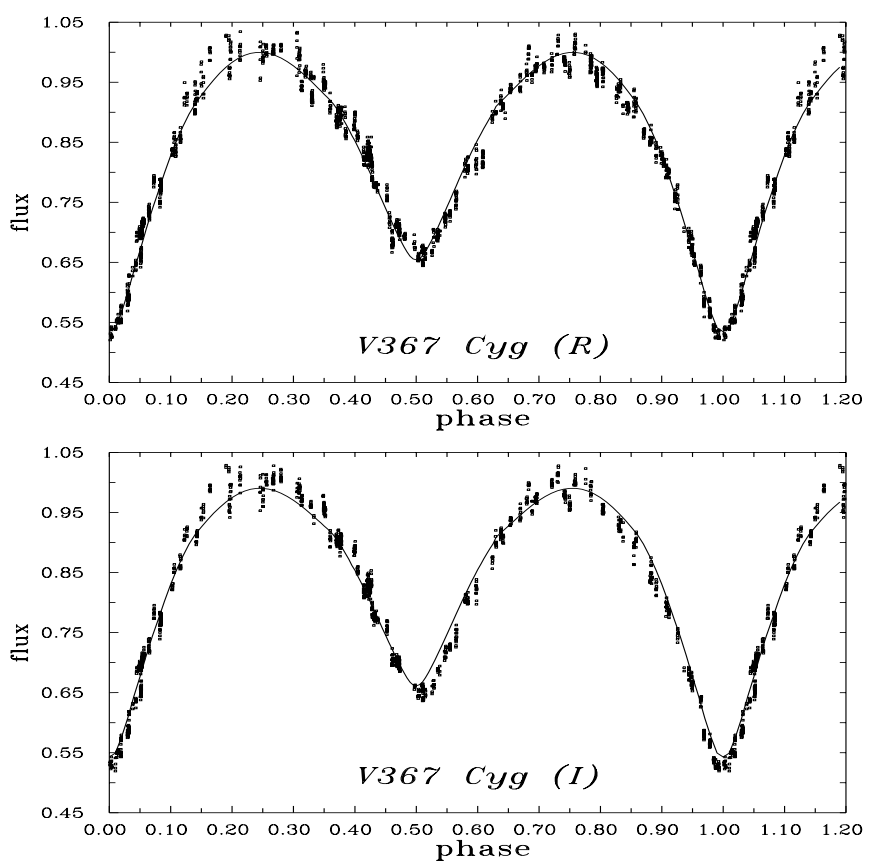

Fig. 3. Comparison between the disk model (lines) and observations (dots) $-R$ and $I$ filters

\subsection{Mass transfer rate}

We have estimated the mass transfer rate in two ways: first, from the outer disk temperature, assuming steady state accretion, and second, from an aproximate formula given by Smak (1989). This was done for V367 Cyg and also for similar, previously analyzed systems: UU Cnc and W Cru. Such calculations gave the results presented in Table 4. Note that the mass transfer rate for W Cru differs from that published by Zoła (1996) due to an error
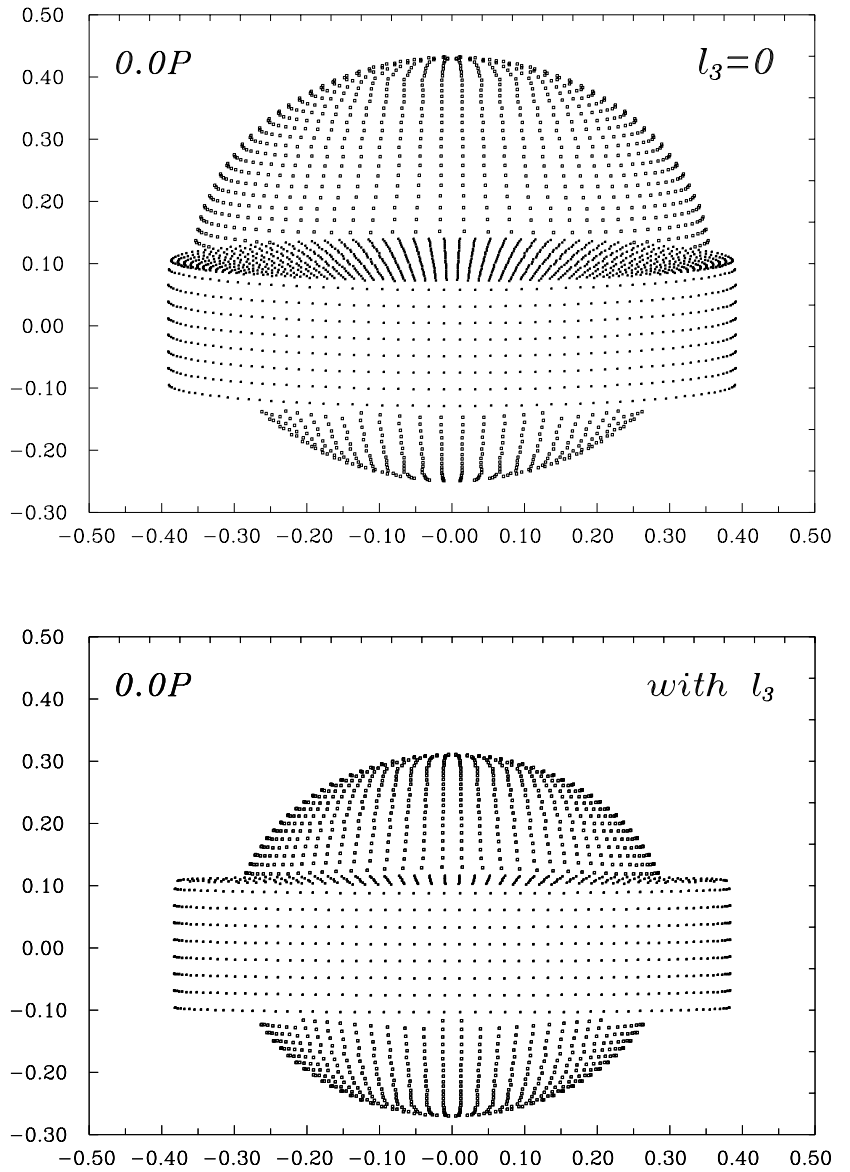

Fig. 4. View of the model of V367 Cyg at phase 0.0 (disk model with and without $l_{3}$ ). Orbital separation units

discovered in the code used for calculation of the maximum disk temperature for the stationary accretion case. These two methods agree well for V367 Cyg but for very large disks (W Cru and UU Cnc) discrepancies are significant.

The mass transfer rate of order of $510^{-5} M_{\odot} / \mathrm{yr}$ in V367 Cyg should produce the following period change: $P^{-1} \mathrm{~d} P / \mathrm{d} t=510^{-5} / \mathrm{yr}$, which is of the same order as that for $\beta$ Lyr. Unfortunately, the $\mathrm{O}-\mathrm{C}$ diagram is not so well covered and the scatter in the $\mathrm{O}-\mathrm{C}$ values is so large (Kreiner 1999, private communication) that no decisive conclusions can be drawn yet. Future, high precision measurements of times of minima are needed to resolve this matter.

\section{Conclusions}

$B, V, R$ and $I$ light curves of V367 Cyg, collected during two consecutive observing seasons, were analyzed with the Wilson-Devinney code (no-disk model) and its modification that also accounts for the presence of an accretion disk (disk model).

Within the no-disk model we derived a contact configuration. The temperature of the primary component was fixed at $8000 \mathrm{~K}$. The secondary's temperature should be about $6500-6700 \mathrm{~K}$. This solution would imply that 
Table 3. Absolute parameters of V367 Cyg for different models

\begin{tabular}{lrrr}
\hline parameter & $\begin{array}{r}\text { no-disk } \\
\text { no } l_{3}\end{array}$ & $\begin{array}{r}\text { disk } \\
\text { with } l_{3}\end{array}$ & $\begin{array}{r}\text { disk } \\
\text { no } l_{3}\end{array}$ \\
\hline$M_{\mathrm{p}}$ & $21.7 \pm 2.8 M_{\odot}$ & $1.10 \pm 0.5 M_{\odot}$ & $3.3 \pm 0.9 M_{\odot}$ \\
$M_{\mathrm{S}}$ & $11.0 \pm 0.8 M_{\odot}$ & $2.5 \pm 0.4 M_{\odot}$ & $4.0 \pm 0.5 M_{\odot}$ \\
$R_{\mathrm{p}}$ & $43.0 \pm 2.7 R_{\odot}$ & $14.2 \pm 1.3 R_{\odot}$ & $21.3 \pm 2.0 R_{\odot}$ \\
$R_{\mathrm{s}}$ & $30.8 R_{\odot}$ & $* 2.3 R_{\odot}$ & $* 2.9 R_{\odot}$ \\
$A$ & $98.0 \pm 6.2 R_{\odot}$ & $46.9 \pm 4.2 R_{\odot}$ & $59.6 \pm 5.5 R_{\odot}$ \\
\hline$R_{\mathrm{d}}$ & & $17.9 \pm 1.1 R_{\odot}$ & $23.3 \pm 2.5 R_{\odot}$ \\
$Z_{\mathrm{d}}$ & & $5.1 \pm 1.2 R_{\odot}$ & $6.4 \pm 1.5 R_{\odot}$ \\
$T_{\mathrm{d}}$ & & $5200 \pm 700 \mathrm{~K}$ & $5300 \pm 1500 \mathrm{~K}$ \\
\hline
\end{tabular}

* - if a Main Sequence star.

V367 Cyg is a massive (total mass about $30 M_{\odot}$ ) contact system. A significant amount of third light is required, ranging from 18 percent in the $I$ filter to almost 23 percent in $B$. It is too low, however, to be due to star $\mathrm{D}$, and much too high if the star $\mathrm{C}$ is the additional contribution (see comments below on the existence of star D). As suggested by the referee, the third light may be just a numerical artifact. It is possible that a spurious solution was obtained due to the use of the wrong model (not accounting for disk effects) for a system with an accretion disk. Due to large disagreement in the third light contribution the no-disk solution with third light was finally discarded.

In our disk model, matter is transferred from the lower mass component to the more massive star which is completely obscured by the accretion disk. The latter result was derived due to our very crude model. We assumed the disk to be optically thick while the real disk structure should have an atmosphere (most likely extended). Our model does not account for the effect of some of the radiation from the mass accreting star passing through the disk atmosphere. Such an effect could be a possible explanation for the observed $10000 \mathrm{~K}$ continuum. We obtained different mass ratios: $q=0.44$ if we included third light and $q=0.83$ for a solution without $l_{3}$. Since there is evidence for a third star in V367 Cyg, the light curve solution should include $l_{3}$. However, this turned out to be a risky procedure, especially in the disk model. As one can see in Fig. 4, the disk is not completely obscured by the primary star and the light from these parts would have the same effect on the light curve as a third light. The amount of third light we obtained is about ten times larger than star C should contribute.

Additional check for consistency of our models can be done with simple calculations of predicted absolute magnitudes of the system which could be compared with that derived from the apparent magnitude, known reddening and distance to V367 Cyg. The Hipparcos parallax is very small $(\pi=0.88$ mas \pm 0.63 mas $)$ indicating a distance of about $1.1 \mathrm{kpc}$ (although with large error: $0.66 \mathrm{kpc}$ to $4 \mathrm{kpc}$ ). The distance derived from the $0.22 \mu \mathrm{m}$ feature in the ultraviolet spectrum is about $0.7 \mathrm{kpc}$ (Elias 1993). The distance of $0.7 \mathrm{kpc}$ will require $M_{\mathrm{v}}=-3.85^{\mathrm{m}}$, while $1.1 \mathrm{kpc} M_{\mathrm{v}}=-4.83^{\mathrm{m}}$ and $4 \mathrm{kpc} M_{\mathrm{v}}=-7.63^{\mathrm{m}}$.
Table 4. Mass transfer rates for V367 Cyg, UU Cnc and W $\mathrm{Cru}$

\begin{tabular}{llrr}
\hline & \multicolumn{4}{c}{$\begin{array}{r}\text { stationary } \\
\text { object }\end{array}$} & data & $510^{-5} M_{\odot} / \mathrm{yr}$ & $710^{-5} M_{\odot} / \mathrm{yr}$ \\
\hline V367 Cyg & ZO & $510^{-7} M_{\odot} / \mathrm{yr}$ & $210^{-9} M_{\odot} / \mathrm{yr}$ \\
UU Cnc & APT & $110^{-7} M_{\odot} / \mathrm{yr}$ & $910^{-9} M_{\odot} / \mathrm{yr}$ \\
UU Cnc & KRK & $510^{-7} M_{\odot} / \mathrm{yr}$ & $510^{-7} M_{\odot} / \mathrm{yr}$ \\
W Cru & Pazzi & $810^{-5} M_{\odot} M_{\odot} / \mathrm{yr}$ \\
W Cru & Marino & $310^{-5} M_{\odot} / \mathrm{yr}$ & $20^{-7} M_{\odot} / \mathrm{yr}$
\end{tabular}

$\mathrm{ZO}$ - this paper, disk solution without $l_{3}$;

APT - APT data (Eaton et al. 1991);

KRK - Cracow data (Winiarski \& Zoła 1987);

Pazzi - Pazzi (1993);

Marino - Marino et al. (1988).

Taking effective temperatures and radii from Table 3 we have: $M_{\mathrm{v}}=-4.98^{\mathrm{m}}$ for the no-disk, no $l_{3}$ solution, $M_{\mathrm{v}}=-3.43^{\mathrm{m}}$ for the disk model without third light and $M_{\mathrm{v}}=-2.60^{\mathrm{m}}$ for the disk model with third light. The disk model with $l_{3}$ gives an absolute magnitude of V367 Cyg defintely too low so we conclude that the disk solution with third light could also be a numerical artifact due to the large number of free parameters. A determination of the star $\mathrm{C}$ magnitudes in different filters independent from light curve modeling (i.e. by CCD observations) would help to resolve the problem of third light and allow more reliable determination of the mass ratio of the system on the basis of light curve modeling. If the distance to V367 Cyg is close to $0.7 \mathrm{kpc}$ the closest match is obtained for the disk model without third light. However if the distance is about $1 \mathrm{kpc}$, the no-disk solution gives almost perfect agreement. Unfortunately, on the basis of the above calculations we cannot distinguish between the no-disk and disk solutions.

For the disk model, the primary component parameters are: mass about $M_{\mathrm{p}}=3.3 M_{\odot}$ and radius about $R_{\mathrm{p}}=21 R_{\odot}$. The disk around the invisible component is large, its radius being about $23 R_{\odot}$, almost completely filling the mass gainer's Roche lobe. The disk luminosity increases from the $B$ to $I$ wavelengths, its contribution reaching about $21 \%$ of the total light in the $I$ filter.

The disk and no-disk models gave a comparable fit to the observed light curves. The best fit was obtained for the disk model with third light. The fits within the no-disk model also gave a good description of the observations, which is not surprising as the light curves of a high inclination system with an accretion disk resemble those of contact systems. As was shown by Zoła (1995), the best fit will likely result in a contact configuration when the Wilson-Devinney model is used to solve light curves of a high-inclination binary system with one component hidden in an optically thick accretion disk.

The latest model for V367 Cyg proposed by TB98 has been verified with our new photometric data. The quality of the fit is worse than that of our disk model. In order to reproduce the observed light curves with the mass ratio 
claimed by Berdyugin \& Tarasov (1997), the disk must either exceed the Roche lobe or be very thick. If the disk radius is limited to the side radius of the secondary star Roche lobe, the $\beta$ parameter needs to be close to 35 degrees. This is not a moderately thick disk and it should be hotter than the primary (visible) component.

Finally, we looked for star D in the photometric data. For this purpose, we made a grid of solutions (within the disk model), with the third light parameter fixed at chosen values. The gradient search method was employed and only the $V$ light curve was used. We set as the starting parameters those derived earlier. The third light parameter was considered in the range from 0 to 0.6 , with step 0.05. It turned out that the quality of the fit deteriorates quickly for increasing third light, being unacceptable already for $l_{3}=0.30$. The conclusion is that there cannot be such a bright third star in V367 Cyg. The inspection of the Hipparcos catalogue confirms this inference - no such star is listed in the Hipparcos catalogue of multiple stars.

Neither the Roche nor disk models can explain the observed asymmetry of the shallower minimum and slightly different heights of maxima. Either a spot should be added to the Roche model or a non circular disk assumed to improve the fits.

Acknowledgements. The computations were performed at ACK "Cyfronet" in Cracow under grant No. KBN/UJ/015/95 which we gratefully acknowledge. The authors thank M. Drożdż, D. Marchev, J. Krzesiński and G. Pajdosz for obtaining some observations. We would like to thank Prof. J. Smak for stimulating discussions and critical reading of the manuscript and Prof. J. Mikołajewska for discovering a bug in the code used to compute the mass transfer rate for $\mathrm{W}$ Cru. We acknowlege comments of an anonymous referee which allowed us to improve presentation of this work.

\section{References}

Akan, M. C. 1987, Ap\&SS, 135, 157

Aydin, C., Hack, M., \& Yilmaz, N. 1978, Ap\&SS, 53, 345

Barone, F., Maceroni, D., Milano, L., \& Russo, G. 1988, A\&A, 197,347

Berdyugin, A. I., \& Tarasov, A. E. 1997, Astr.Zhn, 74, 230

Claret, A., Díaz-Cordovés, J., \& Gimenez, A. 1995, A\&AS, 114,247
Díaz-Cordovés, J., Claret, A., \& Gimenez, A. 1995, A\&AS, 110,329

Eaton, J. A., Hall, D. S., \& Honeycutt, R. K. 1991, ApJ, 376, 289

Elias, N. M. 1993, ApJ, 410, 785

Fresa, A. 1966, Mem. Soc. Astron. Ital., 37, 607

Glazunova, L. V., \& Menchenkova, E. V. 1989, SvAL, 15, 107

Hack, M., Engin, S., \& Yilmaz, N. 1984, A\&A, 131, 147

Harmanec, P. 1988, Bull. Astron. Inst. Czech., 39, 329

Heiser, A. M. 1961, ApJ, 134, 568

Heiser, A. M. 1962, ApJ, 135, 78

Kalw, P., \& Pustylnik, I. 1975, Publ. Tartu Astrofiz. Obs., 43, 114

Kreiner, J. M., Krzesiński, J., Pokrzywka, B., et al. 1993, Proc. of the IAU Coll., 136, ed. J. Elliot, \& C. J. Butler (Dublin), 80

Kurucz, R. L. 1979, ApJS, 40, 1

Marino, B. F., Walker, S. G., \& Budding, E. 1988, Ap\&SS, 150, 103

McAlister, H. A., \& Hattkopf, W. I. 1988, The Second catalog of Interferomtric Measurements of Binary Stars (Atlanta: Georgia State Univ. Press), 117

Li, Y.-F., \& Leung, K.-C. 1987, ApJ, 313, 801

Paczyński, B. 1971, ARA\&A, 8, 183

Pazzi, L., 1993, IBVS, 3847

Pavlovski, K., Schneider, H., \& Akan, M. C. 1992, A\&A, 258, 329

Price, W. L. 1976, Computer J., 20, 367

Popper, D. M. 1981, ARA\&A, 18, 115

Schneider, H., Pavlovski, K., Planinić, M., \& Ivezić, Z. 1993, A\&A, 277, 480

Smak, J. 1984, Acta Astron., 34, 161

Smak, J. 1989, SSRv., 55, 107

Smak, J. 1992, in Proc. IAU Symp. No 151, Evolutionary Processes in Interacting Binary Stars, Cordoba, Argentina, 83

Tarasov, A. E., \& Berdyugin, A. I. 1998, Astron. Rep., 42, 494

Wilson, R. E. 1993, Documentation of Eclipsing Binary Computer Model

Wilson, R. E., \& Devinney, E. J. 1971, ApJ, 166, 605

Winiarski, M., \& Zoła, S. 1987, Acta Astr., 37, 375

Zoła, S. 1991, Acta Astron., 41, 213

Zoła, S. 1992, Acta Astron., 42, 355

Zoła, S. 1995, A\&A, 294, 252

Zoła, S. 1996, A\&A, 308, 785

Zoła, S., Kolonko, M., \& Szczech, M. 1997, A\&A, 324, 1010 